\title{
Les approches économiques de la précaution : présentation et discussion critique
}

\author{
Christian Gollier ${ }^{1}$, Nicolas Treich ${ }^{2}$ \\ 1 Économiste, Toulouse School of Economics, 31000 Toulouse, France \\ 2 Économiste, Toulouse School of Economics (LERNA, INRA), 31000 Toulouse, France
}

Le principe de précaution, inscrit dans la Constitution française, a été souvent traité dans NSS avec des points de vue de multiples disciplines et des approches interdisciplinaires. Il est encore l'objet de nombreux débats dans différentes sphères (chercheurs, décideurs, spécialistes des médias, associations, etc.).

L'article de C. Gollier et N. Treich aborde le principe de précaution en se basant sur la science économique. Il en présente une approche séquentielle où la décision s'adapte à l'arrivée de nouvelles connaissances scientifiques. Mais il présente aussi une approche plus novatrice, "le modèle d'aversion à l'ambiguïté », motivée par des expériences en laboratoire et visant à intégrer les doutes sur les croyances. Fruit de travaux associant mathématiciens, psychologues et économistes, cette nouvelle approche peut paraître plus adaptée pour capter les situations d'incertitude forte, mais soulève des questions relatives à la rationalité des décisions qu'elle engendre.

La Rédaction

\section{Mots-clés :} principedeprécaution; économie ; espérance d'utilité ; aversion à l'ambiguïté

\section{Keywords:} precautionary principle; economics; expected utility; belief revision; ambiguity aversion
Résumé - Le principe de précaution est très populaire aujourd'hui. Mais a-t-il un fondement économique ? Nous présentons deux théories économiques de la précaution. La première se base sur le cadre classique d'espérance d'utilité avec révision bayésienne des croyances. La seconde se fonde sur l'aversion à l'ambiguïté. Nous discutons l'intérêt, mais aussi les limites de ces deux approches.

\section{Introduction}

\section{Éléments de contexte}

Depuis la révolution des Lumières, nous exigeons que nos décisions collectives soient conduites par des principes cohérents avec la science. Néanmoins, dans bien des domaines, la science est incertaine, avec de nombreuses zones d'ombre. Elles laissent les décideurs et l'opinion publique dans un certain désarroi. On n'a pas besoin des climatosceptiques pour reconnaître que la science du climat ne nous donne pas encore une image définitive du risque de changements globaux liés à l'augmentation de concentration de $\mathrm{CO}_{2}$. Mais d'autres grandes peurs hantent aussi nos choix collectifs face aux innovations scientifiques de notre temps: nanotechnologies, organismes génétiquement modifiés, ondes électromagnétiques, etc.

Le principe de précaution définit une attitude générale à adopter face à ces risques émergents. On dit souvent qu'il a pour origine le principe allemand de «Vorsorge » (précaution). Une définition du principe de précaution est donnée dans le principe 15 de la Déclaration de Rio sur l'environnement et le développement en

Auteur correspondant : N. Treich, ntreich@toulouse.inra.fr 
1992 ${ }^{1}$. Des définitions proches existent dans le droit national et international. En février 2005, le Parlement français inscrit le principe de précaution dans la Constitution. L'article 5 stipule : «Lorsque la réalisation d'un dommage, bien qu'incertaine en l'état des connaissances scientifiques, pourrait affecter de manière grave et irréversible l'environnement, les autorités publiques veilleront, par application du principe de précaution, et dans leurs domaines d'attribution, à la mise en œuvre de procédures d'évaluation des risques et à l'adoption de mesures provisoires et proportionnées afin de parer à la réalisation du dommage. »

Ainsi, le principe de précaution est présent en France au niveau juridique le plus élevé. L'élément nouveau qu'il introduit est le rapport entre décision et incertitude scientifique. L'ambition de la précaution est en effet de rejeter l'idée que l'incertitude scientifique justifie l'inaction. Il donnerait au contraire aux décideurs le pouvoir de prendre des mesures anticipées malgré l'incertitude scientifique, c'est-à-dire malgré la perspective de progrès de la connaissance. Au début des années 1980, plusieurs traités internationaux ont mis en œuvre des mesures de précaution, à l'image du traité de 1987 sur l'interdiction de rejet de substances toxiques dans la mer du Nord. Le texte de la Commission européenne (2000) se réfère explicitement à l'approche économique quand il interprète le principe de précaution; il stipule en effet que les mesures fondées sur le principe de précaution devraient «être basées sur un examen des avantages et des charges potentiels de l'action ou de l'absence d'action (y compris, le cas échéant et dans la mesure du possible, une analyse de rentabilité économique) ».

\section{Précaution et analyse coût-bénéfice}

Pour réfléchir à la justification économique de la précaution, il semble assez naturel, comme le suggère l'approche de la Commission européenne (2000), de se demander si les dépenses engagées pour réduire un risque sont justifiées au regard des bénéfices que cette réduction procure. Des études empiriques suggèrent que ce n'est pas toujours le cas. Sunstein (2005), par exemple, en comparant les coûts par vie sauvée de différents programmes de réduction de risque aux États-Unis, a montré que les programmes qui concernent les risques de précaution ont tendance à être les plus inefficaces. Autrement dit, nous pourrions sauver plus de vies à un moindre coût en investissant dans la réduction des risques mieux connus et plus familiers, comme la sécurité routière, les risques d'accident au travail ou la prévention précoce des risques de santé.

\footnotetext{
1 Le texte stipule : "Where there are threats of serious or irreversible damage, lack of full scientific certainty shall not be used as a reason for postponing cost-effective measures to prevent environmental degradation. »
}

Mais comment peut-on comparer des coûts financiers à des bénéfices en matière de réduction de risque de mortalité ou de santé? L'approche économique propose, pour évaluer et comparer les coûts et les bénéfices de tout programme public, de se baser sur les préférences révélées par les choix effectifs des citoyens. L'idée est que l'étude de nos choix privés dans des situations à risque donne des informations sur notre consentement à payer pour la réduction de risque. Ce consentement à payer indique la valeur monétaire que nous attribuons à la réduction de risque, ce qui permet de comparer le bénéfice en " équivalent monétaire » de réduire le risque au coût financier de l'effort de réduction. Par exemple, si nous choisissons d'acheter un véhicule plus cher parce qu'équipé de l'ABS plutôt que de nous offrir un week-end au soleil, nous révélons une information sur la valeur que nous accordons à la préservation de notre sécurité $^{2}$. L'approche économique de la prévention des risques collectifs est basée sur l'idée que la société doit valoriser les avantages de cette prévention à l'aulne des valeurs que les citoyens accordent à ses effets. Elle est fondamentalement citoyenne, elle rejette le paternalisme.

La justification de la précaution se fonde ainsi sur l'étude du comportement individuel en situation d'incertitude. Mais en économie on ne comprend les comportements qu'en les modélisant, et en testant scrupuleusement les modèles en utilisant les données de terrain. Ces données proviennent de l'observation des décisions individuelles sur les différents marchés du risque (par exemple les investissements financiers, la demande d'assurance, les achats de biens de consommation permettant d'augmenter la sécurité), mais elles peuvent provenir aussi de jeux expérimentaux rémunérés en laboratoire ou bien d'enquêtes. La suite de l'article présente les deux modèles de décision dominants en économie qui permettent de représenter les comportements individuels en situation d'incertitude scientifique.

\section{Le modèle d'espérance d'utilité}

\section{Décision face au risque et arrivée d'information}

Le premier modèle est le plus classique; il est basé sur la théorie d'espérance d'utilité (EU) de von Neumann et Morgernstern, introduite par Daniel Bernoulli au $\mathrm{XVIII}^{\mathrm{e}}$ siècle ${ }^{3}$. Pour présenter ce modèle, il est utile de partir d'un exemple simple. Considérons un jeu où un individu fait face à une urne A qui contient 10 boules : 5 blanches et

\footnotetext{
2 Il existe en particulier beaucoup de travaux sur la relation risque-salaire, estimée économétriquement à partir de données sur la mortalité sur le lieu de travail. Voir par exemple Viscusi et Aldy (2003) pour une méta-analyse.

3 Pour une traduction en anglais, voir Bernoulli (1954).
} 


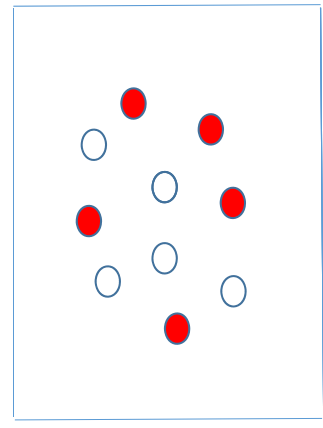

Urne A (" risquée ") : $n$ boules rouges 10 - $n$ boules blanches avec $\mathrm{n}=5$

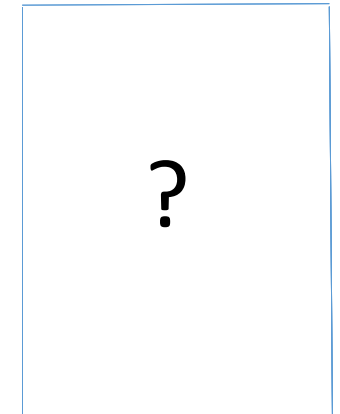

Urne B (" ambigüe ») : $\mathrm{n}$ boules rouges $10-\mathrm{n}$ boules blanches avec $n$ inconnu

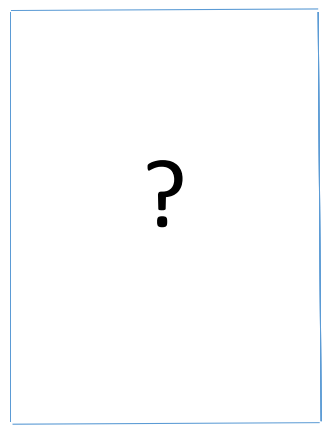

Urne C (" composée ») : $n$ boules rouges 10 - $n$ boules blanches avec $n$ suivant une loi uniforme discrète sur $\{0, \ldots, 10\}$

Fig. 1. Urnes A, B et C.

5 rouges (voir l'urne A sur la Figure 1). On lui annonce qu'il recevra 100 euros si la boule rouge est tirée de l'urne A. Quel est le consentement à payer $S$ de cet individu pour participer à ce jeu ? Dans sa forme sans aversion au risque, la théorie de l'EU nous dit que le consentement à payer est égal à l'espérance de gain du jeu, c'est-à-dire $S=100 \times 0,5=50$ euros. On dit dans ce cas que l'individu est neutre au risque. Plus généralement, cette théorie permet l'aversion au risque impliquant un consentement à payer inférieur à l'espérance de gain. Comme ce trait de préférence de l'être humain n'est pas central pour la suite, nous simplifions l'analyse en supposant la neutralité au risque (pour une introduction à l'économie du risque, voir par exemple Eeckhoudt et al., 2005).

Mais comment introduire de l'incertitude scientifique dans ce jeu? Une première étape consiste à relâcher l'hypothèse que la probabilité de gain - c'est-à-dire la proportion des boules rouges et blanches - est connue. Par exemple, on pourrait envisager une situation où le joueur fait face à une urne $B$, pour laquelle il ignore cette proportion (voir l'urne B sur la Figure 1). Cette ignorance représente en quelque sorte le manque de "connaissances scientifiques » sur le processus qui aboutit au tirage d'une boule. Savage (1954) a généralisé la théorie de l'EU à des situations de ce type. La théorie de Savage suppose que l'individu construit mentalement une probabilité subjective $p$ de gagner, et calcule sa stratégie optimale en maximisant son espérance d'utilité fondée sur cette probabilité subjective. Dans ce cas, un joueur neutre au risque serait simplement prêt à payer $S=100 \times p$ euros.

Un intérêt majeur du modèle d'EU est qu'il peut être facilement généralisé à une perspective séquentielle, qui intègre les progrès de la connaissance. Reprenons encore le jeu précédent, mais en l'adaptant. Considérons maintenant une urne $\mathrm{C}$ dans laquelle le nombre de boules rouges n'est pas connu, mais le joueur pense qu'il y a autant de chances qu'il y ait exactement $0,1, \ldots, 10$ boules rouges dans l'urne (voir l'urne C sur la Figure 1). Dans ce cas, on peut facilement distinguer deux situations : i) le joueur n'apprendra jamais la vraie proportion, ce qui ramène au cas de l'urne A puisque le joueur pense qu'il y a une probabilité de $50 \%$ de tirer une boule rouge, ii) le joueur va recevoir un signal l'informant sur la vraie distribution de probabilité. Par exemple, le joueur pourrait être autorisé à faire des "expériences", au sens où il pourrait simuler quelques tirages et observer la couleur de la boule tirée à chaque tirage. Cette idée de signal basée sur des expériences est fondamentale dans la théorie économique classique de la précaution: le signal représente de manière statistique le processus d'acquisition d'information au cours du temps, c'est-à-dire les progrès de la connaissance scientifique.

Ce processus d'acquisition d'information est traditionnellement fondé sur la règle bayésienne de révision de croyances. Muni du modèle d'EU et de cette règle, on peut construire un modèle de choix séquentiel. Ce modèle permet alors d'étudier une question majeure à l'origine des débats sur la précaution : faut-il attendre les progrès de la connaissance scientifique avant d'agir, ou faut-il agir dès maintenant tout en anticipant un progrès de la connaissance à venir ? Il serait trop long et trop technique de détailler les résultats des modèles microéconomiques qui visent à répondre à cette question (voir par exemple Gollier et al., 2000) ; mais signalons que ces résultats ont mis en évidence la tension entre deux effets. 
D'une part, il y a un désir de reporter les efforts de réduction de risque, ce qui permet d'adapter l'effort à la gravité du dommage. C'est un effet de valeur de l'information, qui va à l'encontre de l'idée de précaution. Mais, il y a aussi un désir d'investir dès maintenant dans la réduction de risque, ce qui permet d'éviter que la situation ne s'aggrave ; cet effet est particulièrement pertinent dans des situations où les dommages ont un caractère irréversible, et c'est cet effet qui est précisément mis en avant par le principe de précaution.

\section{Risque versus incertitude}

Depuis Knight (1921), il est courant de distinguer le risque, qui est caractérisé par une loi de probabilité « objective » fondée sur la réalisation d'événements aléatoires ayant une réalité physique bien connue, et l'incertitude, qui ne repose sur aucune base précise d'information. Et de manière très schématique, il est alors usuel d'associer le risque à la prévention et l'incertitude à la précaution. La théorie de Savage n'est pas compatible avec cette distinction. En effet, il n'y a pas de probabilité objective dans la théorie de Savage. Autrement dit, « les probabilités n'existent pas », selon la célèbre expression de De Finetti. Cette expression signifie que les probabilités objectives n'existent pas. Les probabilités sont toujours subjectives, au sens où elles ne représentent que les croyances subjectives des agents, et ne peuvent être (partiellement) révélées que par les actions de ces agents, mais pas observées directement. Ainsi, il n'y a pas de distinction risque/incertitude au sens classique de la théorie de la décision bayésienne.

Cependant, en se fondant sur l'approche ci-dessus, on peut néanmoins proposer une distinction risque/ incertitude. Le risque serait défini comme une situation où la probabilité subjective n'évolue pas, comme c'est le cas de l'urne A. Par contraste, l'incertitude serait alors définie comme une situation où la probabilité subjective peut évoluer au cours du temps. Reprenant l'exemple de l'urne C, la probabilité subjective sera potentiellement révisée dans le futur en fonction du signal qui sera reçu sur la composition de l'urne. Ainsi, quand le principe de précaution nous parle de "l'état des connaissances scientifiques », on est typiquement dans une situation d'incertitude puisque les croyances peuvent évoluer au rythme des progrès scientifiques. Par conséquent, on pourrait dire que la prévention est relative à la gestion du risque tandis que la précaution est relative à la gestion de l'attente d'information.

Dans des contextes évolutifs, il est donc crucial de définir des méthodes de gestion de risque profondément dynamiques, qui intègrent l'évolution des connaissances. Comme l'écrit la Commission européenne (2000), les «mesures basées sur le principe de précaution doivent être réexaminées à la lumière des nouvelles données scientifiques ». L'analyse coût-bénéfice des décisions publiques face aux risques de précaution doit donc intégrer cette perspective temporelle avec évolution des connaissances. Le concept-clé ici est alors celui de «valeur d'option ». Ce concept indique qu'il existe une valeur à maintenir des degrés de flexibilité au cours du temps afin d'ajuster la décision future aux nouvelles informations. Cette valeur doit être incorporée dans l'analyse coût-bénéfice des différentes actions de gestion de risque.

Pourtant, signalons qu'il est difficile en pratique de calculer des valeurs d'option. Ce calcul requiert non seulement de représenter l'incertitude par des distributions de probabilité, mais aussi de spécifier le timing de résolution de l'incertitude au cours du temps. Ainsi, comme le reconnaît par exemple le guide méthodologique sur l'analyse coût-bénéfice de Pearce et al. (2006), les exemples concrets de calcul de valeur d'option sont à ce jour très limités. Citons néanmoins la littérature sur les options réelles qui utilisent des techniques financières pour calculer des valeurs d'option (voir par exemple Dixit et Pindyck, 1994) ; cette littérature a été appliquée par exemple aux décisions de fermeture/réouverture de ressources minières ou de maintien en activité de centrales nucléaires. Un autre exemple de calcul de valeurs d'option est contenu dans le livre de Nordhaus (1994), dans le célèbre modèle intégré de changement climatique, appelé DICE. Nordhaus développe en effet une analyse de Monte Carlo pour calculer le timing optimal de réduction des émissions de $\mathrm{CO}_{2}$, compte tenu d'hypothèses différentes sur la date à laquelle tous les paramètres du modèle DICE seront effectivement connus.

\section{Le modèle d'aversion à l'ambiguïté}

\section{Le choix d'Ellsberg}

Keynes remarquait que si deux situations sont équivalentes en ce qui concerne leurs distributions de probabilités subjectives, on devrait préférer celle qui est basée sur une somme de connaissance plus élevée. La théorie de l'EU de Savage apparaît souvent comme incompatible avec cette idée. En effet, il n'y a pas de notion de confiance dans une probabilité dans la théorie de Savage. La théorie de l'EU est telle que le décideur se comporte « comme si » il n'y avait toujours qu'une et une seule distribution de probabilités. Dans l'exemple de l'urne B, le joueur assigne une probabilité $p$; celle-ci est unique, et si le joueur faisait face à une urne avec une probabilité « objective » $p$, la situation serait strictement équivalente pour lui, et son consentement à payer serait le même. Pour illustrer, prenons un autre exemple, plus concret : la Figure 2 représente les distributions de probabilité 


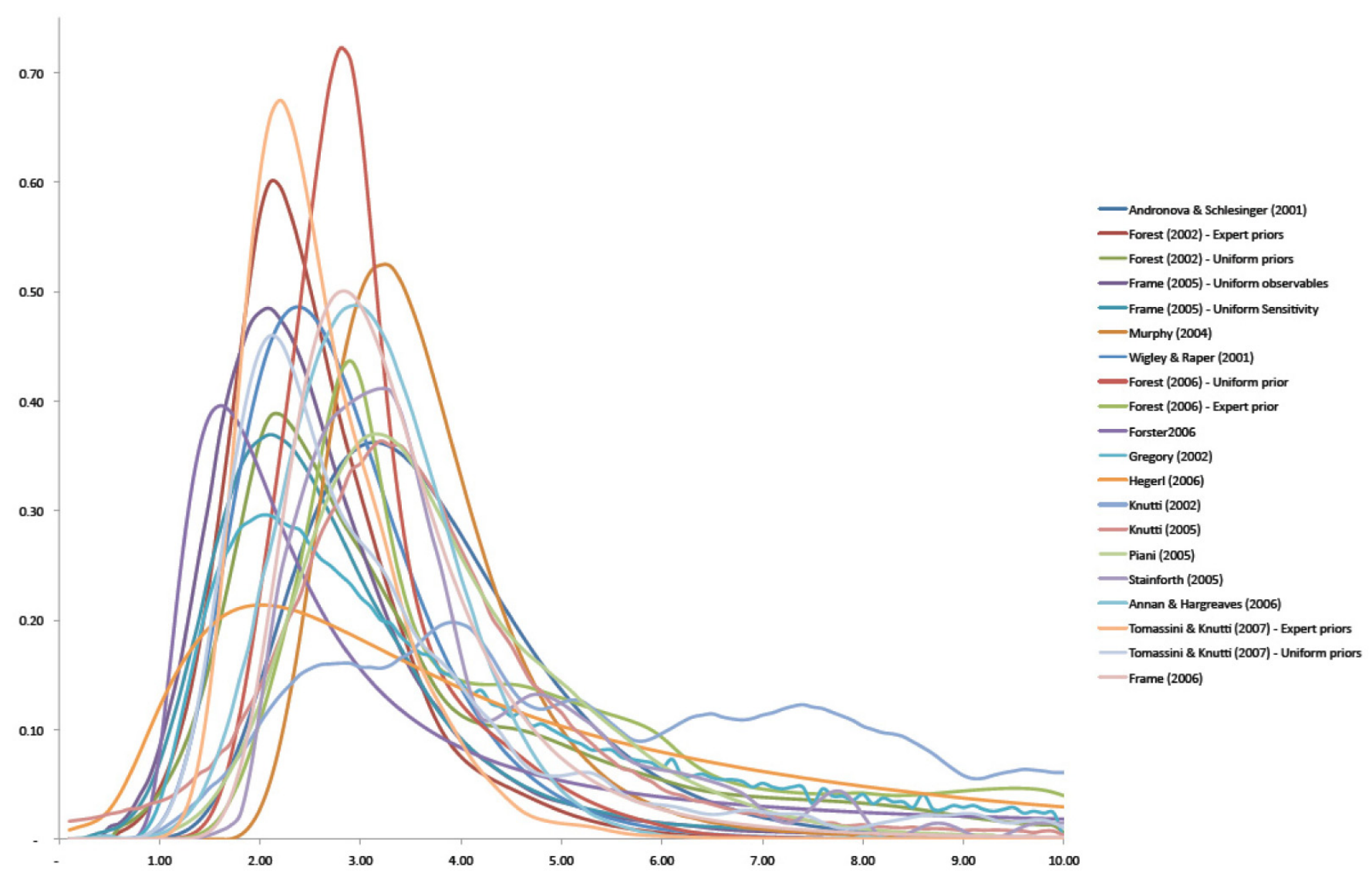

Fig. 2. Différentes distributions de probabilité pour le paramètre de sensibilité du climat (source : Meinshausen et al., 2009).

relatives au paramètre de sensibilité du climat ${ }^{4}$. Peut-on, comme le postule le modèle de Savage, réduire cette situation à une distribution de probabilité unique?

Cette hypothèse de probabilité subjective unique de Savage n'est pas compatible avec un grand nombre d'observations issues des jeux expérimentaux en laboratoire. C'est Ellsberg (1961) le premier qui a suggéré de faire des expériences pour tester l'hypothèse de Savage. Dans une de ses expériences, Ellsberg suggère de proposer au joueur de choisir entre l'urne A et l'urne B, mais en lui laissant aussi choisir la couleur de la boule tirée. La prédiction de la théorie de Savage sur le comportement à adopter est claire: le joueur devrait toujours choisir l'urne B. En effet, soit il pense que la probabilité subjective de tirer une boule rouge dans l'urne B est défavorable $p<0,5$, et il devrait choisir l'urne B en pariant sur le tirage d'une boule blanche ; soit il pense que cette probabilité est favorable $p>0,5$, et il devrait encore choisir l'urne $B$, mais en pariant sur le tirage d'une boule rouge.

\footnotetext{
4 Le paramètre de sensibilité du climat indique le degré de réchauffement de la température pour un doublement de la concentration de $\mathrm{CO}_{2}$ dans l'atmosphère.
}

Dans les deux cas, il devrait donc préférer strictement l'urne B à l'urne A. Seulement dans le cas $p=0,5$, il est exactement indifférent de jouer avec l'urne A ou l'urne B. Pourtant, les études expérimentales de l'expérience de pensée d'Ellsberg ont montré que les joueurs préfèrent jouer avec l'urne A ; c'est le paradoxe d'Ellsberg.

L'argument le plus souvent avancé pour expliquer le paradoxe d'Ellsberg est que nos choix expriment une forme d'aversion à l'ambiguïté sur les probabilités. Autrement dit, les joueurs préfèrent éviter de parier sur l'urne B car les probabilités y sont ambigües. Ils préfèrent l'urne A où les probabilités sont connues. Gilboa et Schmeidler (1989) ont formalisé cette idée avec la théorie $\mathrm{du}$ « maxmin ». Selon cette théorie, face à une situation d'ambiguïté sur les probabilités, le joueur forme non plus une probabilité subjective unique, mais un ensemble de probabilités subjectives. Ensuite le joueur prend sa décision par rapport à la pire des probabilités dans cet ensemble, d'où le terme maxmin. En d'autres termes, le joueur est « catastrophiste », dans le sens où il pense que la probabilité du plus mauvais état est la seule à considérer parmi celles qui lui paraissent plausibles. Supposons par exemple qu'il pense que la proportion de boules 
rouges est comprise entre $1 / 5$ et $4 / 5$ (autrement dit, il pense qu'il y a au moins deux boules de chaque couleur dans l'urne). Dans ce cas, s'il parie sur une boule rouge, il évaluera cette loterie comme s'il n'y avait que $1 / 5$ de boules rouges. Au contraire, s'il parie sur une boule blanche, il évaluera aussi cette loterie comme s'il n'y avait que $1 / 5$ de boules blanches. On est en présence d'une théorie où la somme des probabilités ne vaut plus nécessairement 1 ! Dans les deux cas, son consentement à payer pour participer au jeu ne sera que de 20 euros (sous neutralité au risque). Entre les urnes A et B, le joueur préfère donc toujours parier sur l'urne $A$, qui a une valeur de 50 euros. Le critère maxmin peut donc expliquer le paradoxe d'Ellsberg, qui a été largement documenté par de multiples expériences en laboratoire. Mais quelles sont les autres conséquences, plus pratiques, de l'aversion à l'ambiguïté ?

Il est important de signaler alors que la littérature empirique sur l'aversion à l'ambiguïté est presque exclusivement une littérature expérimentale. En effet, il n'existe pas à notre connaissance d'étude de l'aversion à l'ambiguïté sur des données de marché. La raison à cela est probablement relative à la difficulté de trouver des mesures de l'ambiguïté en dehors du laboratoire. En effet, si les expérimentalistes (pour la plupart économistes ou psychologues) peuvent «créer » artificiellement dans le laboratoire des situations où les probabilités sont présentées comme objectives, et d'autres comme inconnues ( $c f$. les urnes A et B de la Figure 1$)^{5}$, de telles situations bien distinctes entre risques objectifs et risques ambigus semblent difficiles à trouver dans la vie réelle.

Néanmoins, les économistes ont étudié théoriquement l'effet de l'aversion à l'ambiguiité dans des modèles de choix. Et même si ces effets n'ont pas encore été validés empiriquement en dehors du laboratoire, ils peuvent potentiellement expliquer des paradoxes que le modèle bayésien pouvait difficilement expliquer auparavant (comme le paradoxe d'Ellsberg). Ainsi, en finance, l'aversion à l'ambiguité pourrait expliquer le paradoxe du « equity home bias » (French et Poterba, 1991), à savoir la tendance à peu investir dans les actifs financiers étrangers malgré les gains possibles relatifs à la diversification, ainsi que le paradoxe de la prime de risque (Mehra et Prescott, 1985), à savoir la tendance à peu investir dans les actifs financiers risqués malgré les rendements

\footnotetext{
5 Notons ici que la littérature expérimentale sur l'ambiguïté fait l'hypothèse de l'existence de probabilités objectives. Néanmoins, cette hypothèse est discutable puisqu'il apparaît impossible de vérifier que les sujets perçoivent les probabilités qui leur sont présentées dans le laboratoire comme objectives. Par ailleurs, la littérature axiomatique fait aussi la même hypothèse puisqu'elle considère généralement un cadre à la AnscombeAumann où les probabilités objectives sont postulées ( $c f$. par exemple l'axiome d'aversion à l'incertitude de Gilboa et Schmeidler, 1989).
}

supérieurs sur le long terme. D'autres travaux théoriques ont identifié les conditions techniques sous lesquelles l'aversion à l'ambiguïté conduit par exemple à surréagir à de mauvaises nouvelles (Epstein et Schneider, 2008), à soumettre des enchères plus basses (Chen et al., 2007), à mieux se coordonner dans des situations stratégiques (Eichberger et Kesley, 2009), à s'assurer plus (Alary et al., 2013), et à investir plus dans la réduction des risques de mortalité (Treich, 2010). De plus, des travaux récents ont montré que l'effet de l'aversion à l'ambiguïté ne renforce pas nécessairement celui de l'aversion au risque (Gollier, 2011).

Mais, une autre question importante se pose : pourquoi serions-nous adverses à l'ambiguité ? Si on reprend le paradoxe d'Ellsberg, on pourrait argumenter que sans information sur la proportion des boules rouges et blanches dans l'urne B, on pourrait simplement lancer une pièce et parier sur rouge si celle-ci tombait sur pile par exemple, ce qui rendrait artificiellement la situation avec l'urne B strictement équivalente à celle avec l'urne A (Raiffa, 1961). Que captent finalement les modèles incorporant une aversion à l'ambiguïté ? ${ }^{6}$ Techniquement, tous ces modèles représentent une forme de paranoïa du décideur. Le décideur adverse à l'ambiguïté se comporte en effet comme si une Nature malveillante changeait les chances de gain en sa défaveur. Autrement dit, une cause possible à l'aversion à l'ambiguïté serait une forme de paranoïa inhérente à notre comportement face à l'incertitude, qui serait d'autant plus forte que la situation est ambigüe. D'autre part, des recherches en psychologie expérimentale ont montré que l'aversion à l'ambiguiité est associée au sentiment de manque de compétence (Heath et Tversky, 1991). Enfin, des recherches récentes en neuroéconomie ont montré que les parties actives du cerveau des sujets varient selon que ces derniers font face à l'urne $\mathrm{A}$ ou à l'urne B. En particulier, Camerer et al. (2007) ont montré que l'amygdale, une partie du cerveau impliquée dans les réponses comportementales associées à la peur et l'anxiété, était plus active quand les sujets faisaient face à l'urne B.

\section{Ambiguïté, rationalité et choix publics}

Paranoïa, incompétence, peur, anxiété : mais est-ce rationnel d'être adverse à l'ambiguïté ? Selon la théorie de Savage, ce n'est pas rationnel. Mais la réponse est délicate car cela dépend de la définition de la rationalité. Les théories d'aversion à l'ambiguiité sont construites sur

\footnotetext{
6 Voir par exemple Etner et al. (2012) pour un survol récent de la littérature sur les modèles d'ambiguïté et d'aversion à l'ambiguïté (Choquet EU, maxmin, alpha maxmin, contrôle robuste, modèle de contraction, etc.), avec une discussion précise des différences entre ces modèles. Un exemple de modèle récent et influent est celui de Klibanoff et al. (2005) qui généralise le modèle maxmin et permet de séparer l'aversion au risque de l'aversion à l'ambiguïté.
} 
d'autres axiomes de rationalité que ceux postulés pour la théorie de l'EU de Savage. Un test empirique possible de la rationalité consiste à vérifier si le paradoxe est robuste à l'introspection. L'idée de ce test est de confronter les sujets à leurs propres choix passés, par exemple en leur présentant des arguments pour ou contre le choix d'Ellsberg, et de les faire rejouer. Or, les quelques expériences existantes qui mettent en œuvre un tel test semblent indiquer que les choix d'Ellsberg semblent relativement robustes (Camerer et Weber, 1992).

Citons cependant une expérience récente de Halevy (2007) aboutissant à une conclusion différente: elle suggère en effet que les choix d'Ellsberg ne sont pas rationnels, mais seraient plutôt dus à une erreur cognitive. L'expérience de Halevy est essentiellement basée sur les urnes A, B et $C$ présentées plus haut. Rappelons que les urnes $A$ et $C$ (sans apprentissage) devraient être perçues comme équivalentes par le joueur, puisque les probabilités de gagner sont identiques. Pourtant, il apparaît d'abord que 86 \% des sujets qui ont participé à l'expérience de Halevy ne sont pas rationnels au sens où ils ne sont pas indifférents entre ces deux urnes A et C. Par ailleurs, $80 \%$ des sujets sont adverses à l'ambiguité au sens où ils préfèrent $\mathrm{A}$ à $\mathrm{B}$, confirmant bien le paradoxe d'Ellsberg. Mais l'observation intéressante est la suivante : conditionnellement à un comportement rationnel, $94 \%$ des sujets font un choix compatible avec la théorie de $l^{\prime} \mathrm{EU}$, et, conditionnellement à un comportement irrationnel, $93 \%$ des sujets jouent le choix d'Ellsberg. Cette expérience indique donc qu'il existe une association très forte entre l'aversion à l'ambiguïté et un choix irrationnel, suggérant que cette association est liée à la complexité du calcul de multiplication des probabilités.

Ce débat sur la rationalité des choix de type Ellsberg est fondamental pour la justification économique de la précaution. En effet, autour des débats sur le principe de précaution, il s'agit de sélectionner une décision publique efficace et cohérente. Or, il apparaît comme difficilement acceptable d'intégrer dans le critère de décision publique des erreurs cognitives, voire des éléments psychologiques tels que la paranoïa. L'approche citoyenne qui se fonde sur l'observation des consentements à payer est-elle finalement tenable sous conditions d'incertitude scientifique ? De plus, il a été montré que les modèles d'aversion à l'ambiguité génèrent systématiquement des incohérences temporelles (Al-Najjar et Weinstein, 2009). Autrement dit, dans ces modèles, il peut être optimal de revenir sur le plan d'action optimal défini dans le passé, même sans l'arrivée de nouvelles informations. Il peut même être optimal d'ignorer l'information (gratuite) dans les modèles avec aversion à l'ambiguité. Clairement, ces propriétés sont difficilement compatibles avec une vision normative des décisions publiques.

\section{Conclusion}

Le principe de précaution est très populaire aujourd'hui. Pourtant, quand un risque nouveau apparaît, on assiste presque toujours à de vifs débats au sujet de son application. Ces débats sont en grande partie liés à des interprétations différentes du principe. Il est assez symptomatique à ce sujet de remarquer que le rapport de la Commission européenne (2000) sur le principe de précaution commençait par la phrase suivante : «Quand et comment utiliser le Principe de Précaution, tant dans $1^{\prime}$ Union Européenne que sur la scène internationale, est une question qui suscite de nombreux débats et donne lieu à des prises de position diverses, et parfois contradictoires. »

Dans ce contexte de débat controversé, nous avons proposé une lecture du principe de précaution exclusivement basée sur la théorie économique. Aujourd'hui, il existe principalement deux théories économiques de la précaution.

L'une est classique: elle se fonde sur le modèle d'espérance d'utilité avec révision bayésienne des croyances au cours du temps. Le principal avantage de cette approche est qu'elle repose sur des bases normatives solides. Le principal inconvénient est qu'elle ne permet pas de capter la notion de (manque de) confiance dans les probabilités, qui apparaît pourtant fondamentale pour appréhender les situations de précaution.

La deuxième théorie se fonde sur la notion $\mathrm{d}^{\prime}$ aversion à l'ambiguïté. Cette notion, contrairement à la théorie de l'espérance d'utilité, permet de capter le degré de confiance dans les probabilités. En revanche, les modèles d'aversion à l'ambiguité présentent des propriétés qui n'apparaissent pas satisfaisantes d'un point de vue normatif. Par exemple, ils génèrent de l'incohérence temporelle, ou une valeur de l'information négative. Enfin, et cela est lié, un problème technique se pose avec les modèles d'aversion à l'ambiguïté : comment définir une règle de révision des croyances quand plusieurs croyances coexistent? En pratique : peut-on imaginer $\mathrm{d}$ 'autres règles de révision des croyances que la règle de Bayes dans un processus public d'évaluation du risque?

Ces questions font actuellement l'objet de recherches intensives en mathématique, en psychologie et en économie. Elles sont d'autant plus fondamentales que toute théorie de la décision étudiant la justification de la précaution doit pouvoir répondre à des enjeux séquentiels 
avec arrivée d'information au cours du temps, comme le stipule la définition même du principe de précaution.

\section{Remerciements}

Les travaux présentés dans cet article ont été soutenus par le programme de recherche ANR «NanoInnov» (action «nano et société ») et par la chaire SCOR-IDEI "Marchés des risques et création de valeur». Nous remercions Éric Marsden, les rapporteurs anonymes de la revue et les participants à l'International Forum on Industrial Safety, l'Institut pour une culture de sécurité industrielle de Toulouse et l'école d'été de Roscoff du réseau M3D en 2010 pour les commentaires.

\section{Références}

Alary, D., Gollier, C., Treich, N., 2013. The effect of ambiguity aversion and self-protection, Economic Journal, 123, 573, 1188-1202, DOI: 10.1111/ecoj.12035.

Al-Najjar, N.I., Weinstein, J., 2009. The ambiguity aversion literature: A critical assessment, Economics and Philosophy, 25, 3, 249-284.

Bernoulli, D., 1954. Exposition of a new theory on the measurement of risk (traduit du latin en anglais par Louise Sommer), Econometrica, 22, 23-36.

Camerer, C., Weber, M., 1992. Recent developments in modelling preferences: Uncertainty and ambiguity, Journal of Risk and Uncertainty, 5, 325-370.

Camerer, C.F., Bhatt, M., Hsu, M., 2007. Neuroeconomics: Illustrated by the study of ambiguity aversion, in Frey, B.S., Stutzer, A. (Eds.), Economics and Psychology: A Promising New Cross-Disciplinary Field, Cambridge (MA), The MIT Press, 113-151.

Chen, Y., Katuscak, P., Ozdenoren, E., 2007. Sealed bid auction with ambiguity: Theory and ambiguity, Journal of Economic Theory, 136, 513-535.

Commission européenne, 2000. Communication sur le recours au Principe de Précaution (online: http:/ / ec.europa.eu/).

Dixit, A.K., Pindyck, R., 1994. Investment under Uncertainty, Princeton, Princeton University Press.

Eeckhoudt, L., Gollier, C., Schlesinger, H., 2005. Economic and Financial Decisions under Risk, Princeton, Princeton University Press.

Eichberger, J., Kesley, D., 2009. Ambiguity and social interaction, Oxford Economic Papers, 61, 2, 355-379.
Ellsberg, D., 1961. Risk, ambiguity, and the Savage axioms, Quarterly Journal of Economics, 75, 4, 643-669.

Epstein, L., Schneider, M., 2008. Ambiguity, information quality and asset pricing, Journal of Finance, 63, 1, 197-228.

Etner, J., Jeleva, M., Tallon, J.-M., 2012. Decision theory under uncertainty, Journal of Economic Surveys, 26, 2, 234-270.

French, K., Poterba, J., 1991, Investor diversification and international equity markets, American Economic Review, 81, 222-226.

Gilboa, I., Schmeidler, D., 1989. Maximin expected utility with non-unique prior, Journal of Mathematical Economics, 18, 141-153.

Gollier, C., 2011. Portfolio choices and asset prices: The comparative statics of ambiguity aversion, The Review of Economic Studies, 78, 4, 1329-1344.

Gollier, C., Jullien, B., Treich, N., 2000. Scientific progress and irreversibility: An economic interpretation of the Precautionary Principle, Journal of Public Economics, 75, 1, 229-253.

Heath, C., Tversky, A., 1991. Preference and belief: Ambiguity and competence in choice under uncertainty, Journal of Risk and Uncertainty, 4, 5-28.

Klibanoff, P., Marinacci, M., Mukerji, S., 2005. A smooth model of decision making under ambiguity, Econometrica, 73, 6, 1849-1892.

Knight, F.H., 1921. Risk, Uncertainty and Profit, New York, A.M. Kelley.

Halevy, Y., 2007. Ellsberg revisited: An experimental study, Econometrica, 75, 503-536.

Mehra, R., Prescott, E.C., 1985. The equity premium: A puzzle, Journal of Monetary Economics, 15, 2, 145-161.

Meinshausen, M., Meinshausen, N., Hare, W., Raper, S.C., Frieler, K., Knutti, R., Frame, D.J., Allen, M.R., 2009. Greenhouse-gas emission targets for limiting global warming to $2^{\circ} \mathrm{C}$, Nature, 458 (7242), 1158-1162.

Nordhaus, W.D., 1994. Managing the Global Commons, Cambrigde (MA), The MIT Press.

Pearce, D., Atkinson, G., Mourato, S., 2006. Cost-Benefit Analysis and the Environment. Recent Developments, Paris, OECD Publishing.

Raiffa, H., 1961. Risk, ambiguity and the Savage axiom: A comment, Quarterly Journal of Economics, 75, 4, 690-694.

Savage, L.J., 1954. The Foundations of Statistics, New York, John Wiley and Sons.

Sunstein, C.R., 2005. Laws of Fear: Beyond the Precautionary Principle, Cambridge (UK), Cambridge University Press.

Treich, N., 2010. The value of a statistical life under ambiguity aversion, Journal of Environmental Economics and Management, 59, 1, 15-26.

Viscusi, W.K., Aldy, J.E., 2003. The value of a statistical life: A critical review of market estimates throughout the world, Journal of Risk and Uncertainty, 27, 1, 5-76.

Reçu le 1er août 2012. Accepté le 9 septembre 2013. 